\title{
Optically switchable molecular device using microsphere based junctions
}

V. Faramarzi, C. Raimondo, F. Reinders, M. Mayor, P. Samorì, and B. Doudin

Citation: Appl. Phys. Lett. 99, 233104 (2011); doi: 10.1063/1.3665940

View online: https://doi.org/10.1063/1.3665940

View Table of Contents: http://aip.scitation.org/toc/apl/99/23

Published by the American Institute of Physics 


\title{
Optically switchable molecular device using microsphere based junctions
}

\author{
V. Faramarzi, ${ }^{1}$ C. Raimondo, ${ }^{2}$ F. Reinders, ${ }^{3}$ M. Mayor, ${ }^{3,4, a)}$ P. Samorì, ${ }^{2, b)}$ and B. Doudin ${ }^{1, c)}$ \\ ${ }^{1}$ Institut de Physique et Chimie des Matériaux de Strasbourg - CNRS 7504, Université de Strasbourg, \\ 23 rue du Loess, BP 43, 67034 Strasbourg, France \\ ${ }^{2}$ ISIS - CNRS 7006, Université de Strasbourg, 8 allée Gaspard Monge, 67000 Strasbourg, France \\ ${ }^{3}$ Department of Chemistry, University of Basel, 19 St. Johannsring, 4056 Basel, Switzerland \\ ${ }^{4}$ Karlsruhe Institute of Technology KIT, Institute for Nanotechnology, P.O. Box 3640, 76021 Karlsruhe, \\ Germany
}

(Received 16 September 2011; accepted 7 November 2011; published online 5 December 2011)

\begin{abstract}
Metallic planar electrodes are bridged using microspheres coated with chemisorbed azobenzene self-assembled monolayers. The circuit exhibits light-induced switching, with reproducibility over $90 \%$, as statistically determined and compared to junctions incorporating photo-insensitive alkanethiol layers. Microsphere interconnects provide direct access to molecular transport properties, with reliability and stability, making multifunctional molecular electronics devices possible. (C) 2011 American Institute of Physics. [doi:10.1063/1.3665940]
\end{abstract}

Electrical transport through single molecules ${ }^{1,2}$ or selfassembled monolayers ${ }^{3}$ is an increasingly important area of research, powered by the need of gaining a fundamental understanding of the physics of charge transport in metalmolecules-metal junctions and by the perspective of combining the advantages of organic electronics with the advances of miniaturization of the semiconductor industry. In this frame, a basic question remains to be addressed: how can one best exploit the intrinsic property of a molecule once it is incorporated between electrodes? A critical test involves the use of molecules that by design are capable of changing their state as a response to an external stimuli such as lightirradiation, temperature or pressure changes, electric field biasing, etc. Nowadays, a great deal of effort is devoted to getting unambiguous access to molecular switching, ${ }^{4}$ notably limited by lack of reversibility, ${ }^{5}$ or molecule-independent phenomena such as the formation of metallic filaments acting as new conductive pathways in voltage switching. ${ }^{6}$

Thus, there is, nowadays, a strong need for robust device constructions, providing access to charge transfer through molecules, in a planar geometry with better reproducibility and time stability in electrical characterizations. The possibility to remote control the junction with external stimuli, such as electrical field for multi-terminals gating purposes or illumination for optoelectronics applications, offers new avenues for the exploitation of multi-functional molecular properties. Metallic nanoparticles have been successfully employed in the last few years as building blocks in electronic devices, since they allow bridging the size gap separating bottom-up and top-down nanofabrication routes. They usually involve metallic $(\mathrm{Au})$ cluster of $3-30 \mathrm{~nm}$ in size, trapped between electrodes, ${ }^{7}$ or self-assembling in 2 -dimensional (2D) networks that are macroscopically electrically addressable., Electrical transport is, however, complicated by Coulomb blockade effects, combined with percolation in a 2D network. It would, therefore, be highly advantageous to design nanoparticles circuits involving a single and larger bridging particle.

\footnotetext{
a)Electronic mail: marcel.mayor@unibas.ch.

${ }^{b)}$ Electronic mail: samori@unistra.fr.

${ }^{c}$ Electronic mail: bdoudin@unistra.fr.
}

Long et al. ${ }^{10}$ recently proposed to use a sphere of size between $100 \mathrm{~nm}$ to a few microns as intermediate size circuit element. The main purpose of our letter is to show unambiguously that this methodology can be applied to light-switchable molecules, by providing a proof-of-principle experiment that gives evidence for photoactive responsive molecular devices.

Electrodes with sub- $100 \mathrm{~nm}$ gap and widths of a few tens of microns were prepared by shadow evaporation, with optical lithography ensuring a good versatility in the choice of channel widths and avoiding sacrificial layers source of measurements artifacts. ${ }^{11}$ The two electrodes making the nanotrench are made of a Ni layer protected by $\mathrm{Au}$ of similar thickness. One electrode is $60 \mathrm{~nm}$ thick, $20 \mathrm{~nm}$ greater than the other electrode, owing to the shadow evaporation process. Janus microspheres (Fig. 1) are obtained by vacuum coating silica particles deposited on glass with $30 \mathrm{~nm}$ of $\mathrm{Ni}$ followed by $30 \mathrm{~nm}$ of Au. ${ }^{12}$ Substrate detachment under ultrasonic agitation is successfully obtained in water, ethanol, and dichloromethane solutions, with ease of processing. Their size allows for optical microscopy visualization for rapid checks. The magnetic trapping procedure proposed by Long et al. ${ }^{10}$ was used to incorporate spheres in the gap. Test experiments without molecular films are reported elsewhere. ${ }^{11}$

Azobenzene-based molecules are well-documented photochromic molecules ${ }^{13}$ with the extended trans configuration switching to a compact cis configuration under UV (365 nm) light irradiation and the thermodynamically stable trans state reversibly being recovered upon either irradiation with visible light or thermally. We used a thiol terminated biphenyl azobenzene molecule (AZO) that forms tightly packed selfassembled monolayers (SAMs) ${ }^{14}$ featuring bi-stable electrical characteristics under illumination when incorporated in vertical junctions. ${ }^{15,16}$

Dodecanethiol molecules (C12) SAMs were used as a check system, in order to unambiguously detect the lightswitching capacity by means of comparison experiments. The Au electrodes were also coated with either C12- or AZO-SAMs to ensure a better interfacial packing between chemically affine groups exposed on the two surfaces and limiting the possible occurrence of metallic (filament) shorts. Samples were patterned over UV transparent optical 
(a)

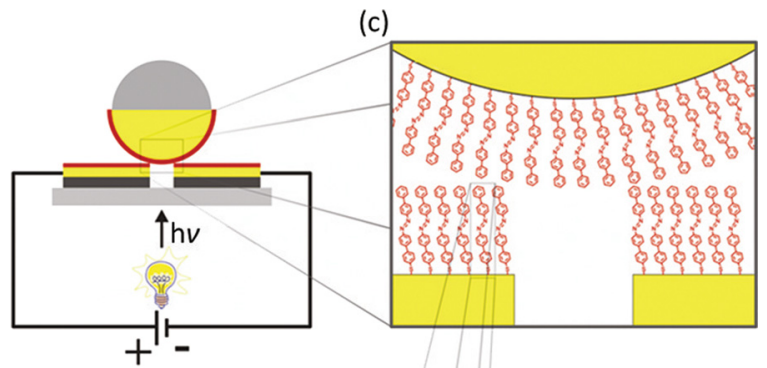

(b)

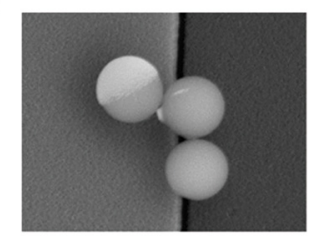

(d)
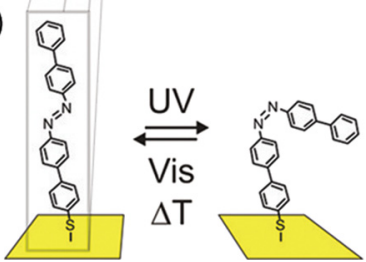

FIG. 1. (Color online) (a) Schematics of the molecular circuit design. The $\mathrm{Au}$ hemisphere of the particle and the electrodes are coated with a chemisorbed C12 or AZO SAMs. (b) Scanning electron microscopy view of $1 \mu \mathrm{m}$ diameter microsphere trapped over an $80 \mathrm{~nm}$ trench. The top left sphere contrast reveals the partial Ni/Au coverage of the spheres. (c) Cartoon of the interaction between molecules in the specific device geometry used. (c) The cis-trans isomerization of the thiol terminated biphenyl azobenzene molecule.

microscopy coverslip glass $(0.17 \mathrm{~mm}$ thick), inserted on an inverted optical microscope platform with an integrated light emitting diode source focused on the sample through the microscope objective (numerical aperture N.A $=0.65$, coverslip substrates), switched between 365 and $455 \mathrm{~nm}$. The illumination power was of the order of $300 \mathrm{~mW} / \mathrm{cm}^{2}$ at $365 \mathrm{~nm}$ and $10 \mathrm{~W} / \mathrm{cm}^{2}$ at $455 \mathrm{~nm}$. For the sake of comparison, another test batch of samples was realized on standard $\mathrm{Si} / \mathrm{SiO}_{2}$ wafer with $550 \mathrm{~nm}$ of oxide preventing electrical shorts. In this case, top illumination was brought through the illuminating condenser (N.A. $=0.55$ ).

A total of 90 nanotrench samples with low leakage currents below $10 \mathrm{pA}$ under $1 \mathrm{~V}$ bias (under illumination) were used. We checked that the leakage current remained small after overnight exposure to typical $0.1 \mathrm{mM}$ solutions. Table I summarizes the statistics over these samples. Their exposure to functionalized spheres resulted in 25 samples categorized as stable closed circuits. Seven among those were categorized as "shorts," with current exceeding $10 \mu \mathrm{A}$ under $1 \mathrm{~V}$ bias that can possibly result from metallic shorts. We also used stringent stability criteria to consider candidates for molecular interconnects: we selected samples exhibiting at least

TABLE I. Statistics of the number of fabricated samples. The type of junction indicates the molecules assembled on the sphere and the electrodes, respectively. The third column counts the number of selected devices after microspheres trapping. The fourth column counts high-currents samples indicating metal shorts.

\begin{tabular}{lcccccc}
\hline \hline $\begin{array}{l}\text { Type of } \\
\text { junction }\end{array}$ & $\begin{array}{c}\text { Fabricated } \\
\text { nanotrenches }\end{array}$ & $\begin{array}{c}\text { Connected } \\
\text { devices }\end{array}$ & $\begin{array}{c}\text { Shorts } \\
\text { Selected }\end{array}$ & $\begin{array}{c}\text { Non } \\
\text { devices }\end{array}$ & Switching \\
switching
\end{tabular}

${ }^{\text {a Single event, irreversible. }}$ two orders of magnitude current increase after microspheres trapping and kept samples showing stable IV curves up to $1 \mathrm{~V}$ stress voltage. Samples categorized as "switching" corresponded to samples where reversible changes of conductance exceeded $10 \%$. Typical observed ratio $R_{\text {trans }} / R_{\text {cis }}$ spanned values between 1.1 and 3 , significantly lower than the values reported in the literature. ${ }^{15,16}$ Example data are shown in Fig. 2, showing successive IV curves after illuminating the sample using 365 and $455 \mathrm{~nm}$ wavelengths. The observed IV curves were rather linear between $\pm 1 \mathrm{~V}$ bias, similarly to previous reports. ${ }^{15,16}$ Non-linearity was observed at larger bias, with possible electrical instabilities. Switching samples exhibited a sequence of conductance properties illustrated by curves 1-3 of Fig. 2. The initial low-conductance value (curve 1) corresponding to the more stable trans state switched to a more conductive state under $365 \mathrm{~nm}$ illumination, interpreted as occurrence of cis configurations (curve 2). Illuminating at $455 \mathrm{~nm}$ revealed the reversibility of the process (curve 3, similar to 1). A total of 18 samples were considered as representative of molecular junctions, with 16 over 18 confirming that azobenzene component of the devices resulted in reversible light-induced switching. We checked on a few samples that the initial trans to cis transition did not occur under $455 \mathrm{~nm}$ excitation. One sample with rather poor conduction $(\mathrm{R} \approx 3 \mathrm{G} \Omega)$ showed a single irreversible switching, even though no AZO molecules were present. We mentioned this sample as "switching," even though reversibility was not found, to emphasize that accidental coincidence can possibly occur when repeating the experiment. We, therefore, conclude from our experiments that we have a good statistical evidence that our devices exhibit changes of conduction related to lightinduced modifications of the molecular films, confirming that the observed resistance value originates from molecular interconnects.

The time evolution of the IV curves (Fig. 3) indicates a time scale of $10^{3} \mathrm{~s}$ to reach the other conduction state value,

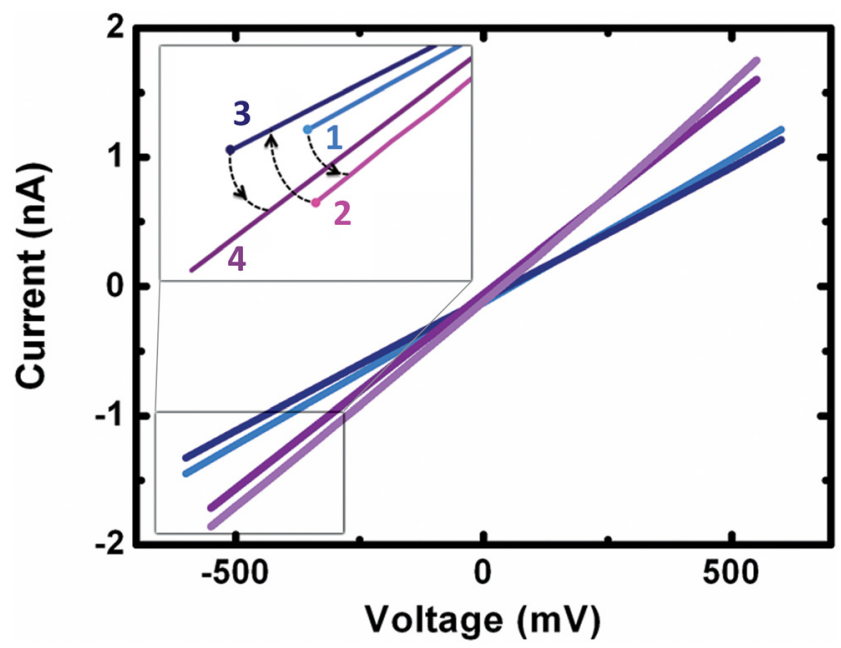

FIG. 2. (Color online) IV curves showing reversible optically modulated current switching in the junction. Curve 1 corresponds to initial junctions, expected to correspond to molecules in trans configuration. Curve 2 results from UV $(365 \mathrm{~nm})$ irradiation, of enhanced conductance due to the occurrence of cis configurations. Curve 3 , after $455 \mathrm{~nm}$ illumination, illustrates the reversibility of the switching, similarly to curve 4 , switching again to $c i s$ after re-illumination with UV. 


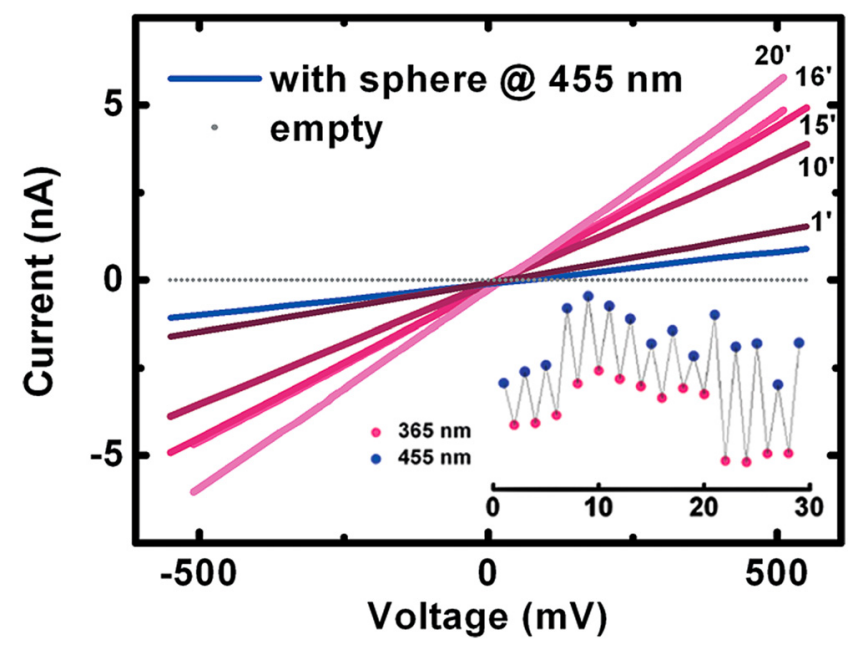

FIG. 3. (Color online) Example of the time evolution of a junction conductance under UV illumination. The functionalized electrodes have a lowcurrent leakage curve (dotted line), with significant increase after trapping functionalized spheres (full line). The other curves show increasing conductivity under increasing indicated illumination time. The inset shows the evolution of the on/off sample resistance after successive UV-visible light illuminations, revealing multiple possible switching events.

in line with other experiments in the vertical geometry. ${ }^{15,16}$ Devices lasted a minimum of several hours, with reproducibility and stability reaching days periods under ambient atmosphere. Up to several tens of switching events can be realized, without any process optimization (Fig. 3 inset). Thus, these findings make possible long-term systematic studies on switching molecular devices.

In conclusion, we showed that functionalized microspheres circuits confirm that the observed electrical transport originates from charges transfer through molecules, with a $90 \%$ confidence ratio. Our proof-of-principle test experiments also indicate that azobenzene-type molecules can be efficiently inserted in a planar circuitry, with on/off ratio smaller than those observed in a vertical geometry. It never- theless confirms unambiguously their interest as reversible photochromic molecular electronics elements, towards multifunctional organic-based electronics, stable over several days timescale under environment atmosphere.

This work was supported by the ITN-SUPERIOR (PITN-GA-2009-238177), FP7 ONE-P large-scale project no. 212311, the NanoSci-E+ projects INTERNET and SENSORS, and the International Center for Frontier Research in Chemistry (FRC). Financial supports by the Swiss National Science Foundation (SNF) and the Swiss Nanoscience Institute (SNI) are also gratefully acknowledged.

${ }^{1}$ N. J. Tao, Nat. Nanotechnol. 1, 173 (2006).

${ }^{2}$ A. Nitzan and M. A. Ratner, Science 300, 1384 (2003).

${ }^{3}$ H. B. Akkerman and B. de Boer, J. Phys.: Condens. Matter 20, 013001 (2008).

${ }^{4}$ B. Feringa, Molecular Switches (Wiley, Weinheim, Germany, 2001).

${ }^{5}$ D. Dulić, S. J. van der Molen, T. Kundernac, H. T. Jonkman, J. J. D. de Jong, T. N. Bowden, J. van Esch, B. L. Feringa, and B. J. van Wees, Phys. Rev. Lett. 91, 207402 (2003).

${ }^{6}$ C. N. Lau, D. R. Stewart, R. S. Williams, and M. Bockrath, Nano Lett. 4, 569 (2004).

${ }^{7}$ T. Dadosh, Y. Gordin, R. Krahne, I. Khivrich, D. Mahalu, V. Frydman, J. Sperling, A. Yacoby, and I. Bar-Joseph, Nature 436, 677 (2005).

${ }^{8}$ J. Liao, L. Bernard, M. Langer, C. Schönenberger, and M. Calame, Adv. Mater. 18, 2444 (2006).

${ }^{9}$ S. J. van der Molen, J. Liao, T. Kudernac, J. Agustsson, L. Bernard, M. Calame, B. van Wees, B. Ferringa, and C. Schönenberger, Nano Lett. 9 , 76 (2009).

${ }^{10}$ D. P. Long, C. H. Patterson, M. H. Moore, D. S. Seferos, G. C. Bazan, and J. G. Kushmerick, Appl. Phys. Lett. 86, 153105 (2005).

${ }^{11}$ J.-F. Dayen, V. Faramarzi, M. Pauly, N. T. Kemp, M. Barbero, B. P. Pichon, H. Majjad, S. Begin-Colin, and B. Doudin, Nanotechnology 21, 335303 (2010).

${ }^{12}$ White-Silica ( sicastar $^{\circledR}$ ) spheres from Micromod GMBH.

${ }^{13}$ N. Tamai and H. Miyasaka, Chem. Rev. 100, 1875 (2000).

${ }^{14}$ G. Pace, V. Ferri, C. Grave, M. Elbing, C. Von Hänisch, M. Zharnikov, M. Mayor, M. A. Rampi, and P. Samorì, Proc. Natl. Acad. Sci. U.S.A. 104, 9937 (2007).

${ }^{15}$ J. M. Mativetsky, G. Pace, M. Elbing, M. A. Rampi, M. Mayor, and P. Samorì, J. Am. Chem. Soc. 130, 9193 (2008).

${ }^{16}$ V. Ferri, M. Elbing, G. Pace, M. D. Dickey, M. Zharnikov, P. Samorì, M. Mayor, and M. A. Rampi, Angew. Chem., Int. Ed. 47, 3407 (2008). 